\title{
Phytosociological Study and Phytoecologique of Psammophytes of the Coastline of The Region of Tlemcen (Oranie-Algeria)
}

\author{
Stambouli-Meziane $\mathrm{H}^{1}$, Merzouk $\mathrm{A}^{1}$ \& Bouazza $\mathrm{M}^{1}$ \\ ${ }^{1}$ Laboratory of Ecology and Management of Natural Ecosystems, Tlemcen, Algeria \\ Correspondence: Stambouli-Meziane H, Laboratory of Ecology and Management of Natural Ecosystems, \\ Tlemcen, Algeria. E-mail: meziane_hassiba@yahoo.fr
}

Received: December 11, 2014 Accepted: December 19, 2014 Online Published: March 11, 2015

doi:10.5539/ijb.v7n2p86

URL: http://dx.doi.org/10.5539/ijb.v7n2p86

\begin{abstract}
Absract
This study is devoted to the analysis of psammophile of coastal dunes of the region of Tlemcen. The interpretation by the factor analysis of matches (A. F. C.) has allowed us to individualize classes' phytosociologique different. The colonize psammophile, par excellence, the embryonic dunes. Some species colonize the dunes vivid. Finally, other occupies the dunes the most advanced and laid down. Using the data and phytosociologique phytodynamiques, we were able to understand the evolution of this vegetation, and its diversity.
\end{abstract}

Keywords: Phytosociologie, psammophile, coastline, tlemcen, Algeria, diversity

\section{Introduction}

The vegetation, the region of Tlemcen, presents a good example of study of plant diversity; and especially an interesting synthesis on the natural dynamics of ecosystems from the shoreline up to the steppe. This study has been launched by several authors. These include mainly: Zeraïa (1981), Dahmani-Megrouche (1997), Quezel (2000), and Bouazza and Benabadji (1998).

The ecosystems Mediterranean coastlines are characterized by climatic constraints and strong soil, salinity, wind, drought and shallow soils or mobile.

The work that we are presenting here concerns the evolution of psammophiles the coastline of the region of Tlemcen. The latter is linked to a high percentage of sand, always higher than $60 \%$. Although they are located in the northern part; in the South, these formations are well represented and are essentially related to the importance of deposits of sand and the presence of gypsum and salts.

This study has been carried out on the basis of the readings phytosociologiques to determine the narrow affinities of different plant groups. In the second place, the knowledge of this floristic richness allows you to make proposals leading to the preservation and improvement of these fragile environments, to limit the degradation and to promote their development in a rational way.

\section{Materials and Methods}

The study covers the analysis of the distribution of species in the the coastal region of Tlemcen: study sites were chosen. From the beach Beni Saf up Marsat Ben M'hidi.

For this we chose two areas repartees as follows:

- Representive areas them live dunes and dune embryonic (from the beach Beni-Saf up to Marsat Ben M'hidi).

- Zones representing the semi-fixed dunes (Ghazaouet cement factory station (Beni-Saf).

The study area is characterized by a high floristic diversity which is related to the combination of ecological factors that are also very varied (variation bioclimatic, Action anthropozoogéne).

This study has been carried out on the basis of the readings phytosociologique to determine the narrow affinities of different plant groups. In the second place, the knowledge of this floristic richness allows you to make proposals leading to the preservation and improvement of these fragile environments, to limit the degradation and to promote their development in a rational way. 
For this study it was selected 10 stations to study locating in the western part of the North West Algeria Figure.1. These are located between $1^{\circ} 27^{\prime}$ and $1^{\circ} 51^{\prime}$ west longitude and $34 \hat{\mathrm{A}}^{\circ} 27^{\prime}$ and $35^{\circ} 18^{\prime}$ north latitude. They are geographically limited:

- $\quad$ to the North by the Mediterranean sea

- $\quad$ to the south by the wilaya of Na'imah

- $\quad$ to the west by the moroccan-algerian border

- $\quad$ to the east by the wilaya of Temouchent

- $\quad$ to the south-east by the wilaya of Sidi Bel Abbes

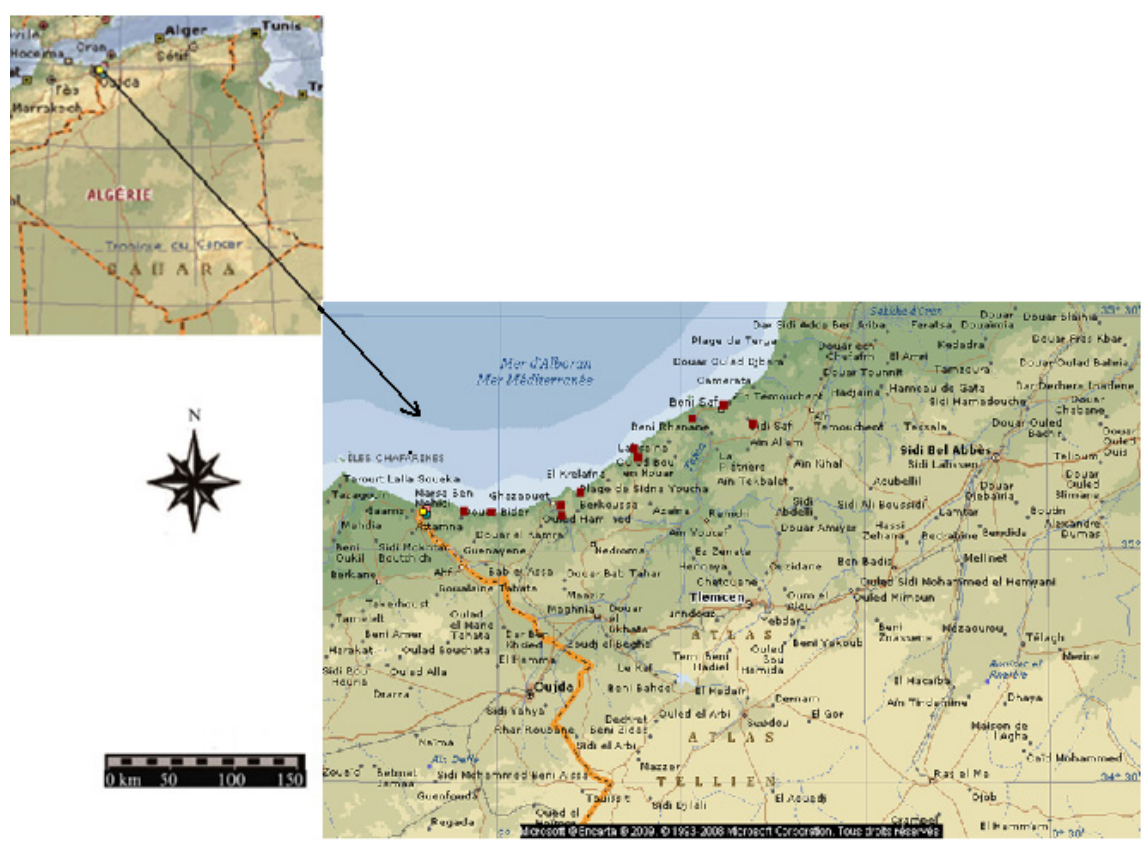

Figure 1. Location of Studies Stations

\subsection{Béni Saf}

Those lands are limestone lithothamniées rich in fossil shells lumachellique of type post- tablecloths Miocene. rest on these limestones intercalations clays to sandstone Tortonian age (Miocene). The limestones constitute a plateau called "Sidi Safi plateau" from which is calcium carbonate noted for cement plant Beni Saf These limestones are covered with places by volcanic formations of type basaltic, Guardia (1975).

\subsection{Rachgoune}

The station is located at the mouth of Tafna. These are the dune deposits at "El Guedim" and, on the right bank of the Oued, in these dunes appear basalt flows black color inter stratified with the volcanic tuffs, Guardia (1975).

\subsection{Genesis of Sea Dunes}

Under the effect of erosion, sand particles are going to move grace to winds to feed the dune ridge of coastline.

\subsection{Dunes}

The wind pushes the sand which will hang on waste brought by the sea. This forms a hump get bigger and bigger.This is the birth of a dune where embryonic going to develop a ephemeral vegetation based on: Medicago marina; Cakile maritima; Euphorbia paralias. According to Favennec (2002), dune is a deposition of sand edified by the wind into coming up against various obstacles such as vegetation and asperities terrain encountered between the beach and the mainland. 
The dynamics of dunes depends on the one hand of the Wind speed and the dimension of sand particles and, on the other hand, obstacles which are the vegetation or the reliefs. As a function of the latter we distinguish 04 kinds of dunes.

- The high dunes: encountered the vicinity of the sea (beach Rachgoune, Beidar, Egla M'Khaled).

- Dunes on slopes: are on slopes exposed to the sea (the valleys Rachgoune).

- Suspended dunes: are formed on the cliffs parallel to neighborhood of the sea (Ouled Ayad).

- Dunes clad: depots constitutes tackles against of the scree of slope. It is characterized by the vegetation based : Crucianella maritima, Thymelaea hirsuta and Elichrysum stoechas. (Marsat Ben M'hidi)

The bioclimatic study for two periods (1913-1938) and (1970-2002). Figure 2 showed a vertical indent of each station in direct relation with the Q2 Emberger. Station Ghazaouet, despite falling on of the value of Q2 always under floor lower semi-arid to hot winter.

This climate favors the extension of vegetation therophytic xerophytes.

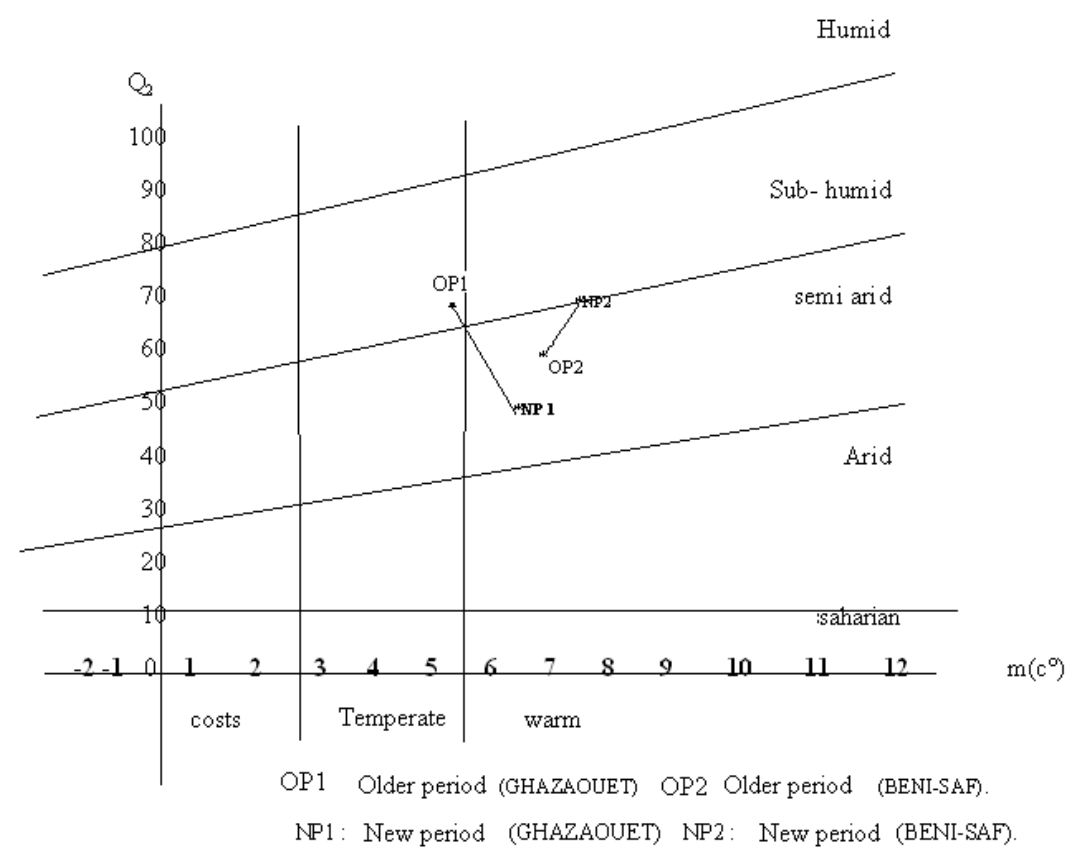

Figure 2. Temperature and humidity within them different zones

\section{Results and Discussion (of the species from the beach)}

This analysis focused on 98 surveys in the beach and shoreline (inside). It subdivided the processing of data in two part that corresponds:

-41 records in the beach

-57 records indoors (coastal)

\begin{tabular}{|l|l|l|l|}
\hline Plan & 1 & 2 & 3 \\
\hline Rate of inertia & 5,5330 & 3,8675 & 3,2664 \\
\hline Eigen values & 0,138 & 0,097 & 0,082 \\
\hline
\end{tabular}

Examination of factorial maps showing the plans of 2/1 and 3/1 projections shows the existence of 04 contrasting sets we will thus attempt to specify what will be the major ecological factors in the diversification of the sward.

\subsection{Plan 2/1:}

The negative side: Lobularia maritima, Chenopodium album, Eryngium maritimum, Asteriscus maritimus, Calycotome spinosa, Chamaerops humilis, Chenopodium album, Hedysarum sp, Juniperus phoenicea 
The positif side: Centaurium unbellatum, Cladanthus arabicus, Juniperus oxycedrus, Paronychia argentea, Rhamnus lycioides, Scabiosa stellata, Spartium junceum, Trifolium stellatum

This axis against indifferent to substrates species and independence vis à vis the water factor.

\subsection{Plan 3/1:}

The negative side: Gnaphalium luteo-album, Lagurus ovatus, Plantago marina, Quercus coccifera, Silene maritima, Spartium junceum, Ulex parviflorus

The positif side: Centaurium unbellatum, Cistus monspeliensis, Cistus salvifolius, Erica multiflora Phagnalon saxatile, Trifolium stellatum

The positive side of this axis lie in particular species characterizing locations silica, and the negative side revealing a less silica than the first pole.

It seems that this group of species in their vast majority is plants that are most commonly seen in the matorral on siliceous substrate to Cisto-Lavanduletea.

In the center of the axis lie in particular a lot of species of Cakile maritima, Ammophila arenaria, Calystegia soldanella, Echinophora spinosa Medicago minima, Medicago marina

These species of psammophytes quintessential (purely psammophilous) that grow on the dunes a strong accumulation Sandy periods of respite from erosion marine; This is a group that is found on the beach of Rechgoune and Marsat Ben M'hidi at an elevation of $0 \mathrm{~m}$ on a low slope of zero and with a very low rate of recovery.

As it moves away from the beach, ecological conditions (Climate, soil) take up the top, and allow the installation of annuals and same perennial basis from: Cynodon dactylon. Silene pseudo-atocion, Lobularia maritima, Teucrium pollium, Matthiola sinuate, Silene conica, Lagurus ovatus, Elichrysum stoechas.

The behavior of species diversity and vegetation stresses the importance of the stability of the substrate for vegetation and already show that the coast is highly structured depending on the distance to the sea. The before dune and dune pioneer are influenced by a strong dynamic of sand and have a low plant collection, as well as a monotonous flora. Greater stability of the sand allows the species to cover larger areas and encourages, Furthermore, the coexistence of most abundant species.

It appears therefore that A.F.C was able to reveal the classic data of the littoral vegetation as described in 1923 by Kuhnholtz-Lordat. In phytosociological terms, maritime dunes belonging to the Ammophilion alliance, characterized by Cakile maritima, Eryngium maritimum et Calystegia soldanella.

Vegetation there forms a complex of plant associations arranged parallel to the shore (Figure 3) and richly described by Molinier and Tallon (1965).

The Agropyretum-mediterraneum is the typical association forming the belt at the base of the dunes (zone 1). Its characteristic species are all observed in (zone 1), we have: Agropyrum junceum, Polygonum maritimum et Cyperus aegyptiacus.

Ammophiletum arundinaceae is the typical of the growing dune (zone 2 and 3). It is characterized by: Ammophila arenaria, Medicago marina et Echinophora spinosa. Enfin, le Crucianelletum settled on the dunes consolidated (area 4 and 5) and is typically: Crucianella maritima, Medicago littoralis et Pancratium maritimum.

The indications of the analysis of vegetation carried out in this study support this vision, but should not consider these associations and their characteristic species too strictly. Indeed, the analyses presented here also highlight the ongoing nature of the changes in vegetation.

Nude beach (zone 0 ) there is no vegetation. This area is in fact continually swept by waves that do allow no plant to set. The sand is so naked up to the larger amplitude wave zone. Furthermore, as the present low amplitude tides Mediterranean, this portion of the beach is never covered by water and does not allow a particular fauna to settle. Apart from his recent tourist interest and the fact that it represents the pulling of the constitutive sand dunes area, this portion of the Mediterranean beach is low biological interest. 
Table 1. Them floristic surveys of the beach and Valleys of Rachgoune

\begin{tabular}{|c|c|c|c|c|c|c|c|c|c|c|c|c|c|c|c|c|}
\hline \multicolumn{17}{|c|}{ Station: valleys of Rachgoune. Beach and Siga } \\
\hline \multicolumn{17}{|l|}{ exposition : north-South } \\
\hline \multicolumn{17}{|l|}{ Recovery : $60-70 \%$} \\
\hline altitude (m) & & 172 & 180 & 205 & 204 & 214 & 206 & 190 & 209 & 212 & 160 & 185 & 180 & 210 & 200 & 160 \\
\hline GENRES SPECIES & Survey & 1 & 2 & 3 & 4 & 5 & 6 & 7 & 8 & 9 & 10 & 11 & 12 & 13 & 14 & 15 \\
\hline Ammophila arenaria (L.) Link. & Poacées & & & & & & & & & & + & & & & & \\
\hline Anagalis arvensis $L$. & Primulacées & 0 & 0 & + & + & & & & & + & & + & & & & \\
\hline Asperula hirsuta $L$. & Rutacées & 0 & 0 & + & + & 1 & 1 & & & & & & + & + & & \\
\hline Asphodelus microcarpus Salzm et Viv. & Liliacées & 0 & 0 & 0 & + & & & & & & & & & & & \\
\hline Avena sterilis $L$. & Poacées & 0 & 0 & 0 & + & & & & & & & + & & & & \\
\hline Bromus rubens $L$. & Poacées & & & & & & & & & & & + & & & & \\
\hline Cakile maritima Scop. & Brassicacées & & & & & & & & & & 1 & & & & & \\
\hline Calendula arvensis $L$. & Astéracées & 3 & 0 & & & & & & & & & & & & & + \\
\hline Calycotome spinosa (L.) Link. & Fabacées & 2 & 2 & 1 & + & 1 & 1 & & & & & & & & & \\
\hline Calystegia soldanella $L$. & Convolvulacées & & & & & & & & & & 1 & & & & & \\
\hline Centaurium umbellatum (Gibb). Beck. & Gentianacées & + & 1 & 2 & + & + & & + & & & & & & & & \\
\hline Chamaerops humilis L. & Palmacées & 0 & 0 & 0 & 1 & & & & & & & & & & & \\
\hline Chrysanthemum grandiflorum (L.) Batt. & Astéracées & & & & & & & & & + & + & + & & & & \\
\hline Chrysanthemum coronarium $L$. & Astéracées & & & & & & & & & & & + & & & & \\
\hline Cistus monspeliensis L. & Cistacées & 2 & 2 & 3 & 4 & 3 & 4 & + & 3 & 3 & 2 & & 1 & 2 & & 1 \\
\hline Cistus salvifolius $L$. & Cistacées & 2 & 2 & 2 & 2 & & + & 1 & & & & & & & & \\
\hline Cladanthus arabicus (L). Cass. & Astéracées & 2 & 2 & 3 & 4 & & 2 & + & + & & & 3 & 1 & 2 & & 3 \\
\hline Cuscuta sp (Tourn). L. & Cuscutacées & & & & & & & & & & & & & & & + \\
\hline Dactylis glomerata L. & Poacées & 1 & 0 & & + & + & & & & & & & & & & \\
\hline Daucus carota $L$. & Apiacées & 2 & 0 & 2 & + & 1 & & & & & & & & & & \\
\hline Daucus carota subsp gummifer Lamk. & Apiacées & & & & & & & & & 1 & + & & & & & \\
\hline Echinops spinosus L. & Apiacées & & & & & & & & & 1 & + & & & & + & \\
\hline Echinophora spinosa $L$. & Apiacées & & & & & & & & & & 1 & & + & & & \\
\hline Echium vulgare Tourn. & Borraginacées & 3 & 0 & & + & & + & & & & & & & + & + & \\
\hline Ephedra fragilis Desf. & Ephedracées & & & & & & & & & 1 & + & & & & & \\
\hline Erica multiflora L. & Ericacées & 1 & 1 & 1 & 2 & 2 & & & & 1 & + & & & & & \\
\hline Euphorbia paralias $L$. & Euphorbiacées & & & & & & & & & 1 & 1 & 1 & & & & \\
\hline Euphorbia peplis $L$. & Euphorbiacées & 0 & 0 & 0 & + & & & & & & & & & & & \\
\hline Fagonia cretica $L$. & Zygophyllacées & 3 & 0 & & & & & & & & & & & & & \\
\hline Gla diolus segetum Ker-Gawl. & Iridacées & & & & & & & & & + & & & & & & \\
\hline Globularia alypum L. & Globulariacées & 2 & 0 & & & + & & & & & & & & & & \\
\hline Gnaphalium luteo-album L. & Astéracées & 0 & 0 & 0 & 0 & + & & & 1 & & & + & & & & 1 \\
\hline Hedysarum sp L. & Fabacées & 2 & 1 & & 2 & & & + & + & & & & & & & 3 \\
\hline Inula crithmoides $L$. & Astéracées & & & + & & & & & + & & & & & & & \\
\hline Juncus maritimus Lamk. & Juncacées & & & & & & & & & 1 & + & & & & & 2 \\
\hline Juniperus oxycedrus $L$. & Cupressacées & 4 & 3 & 4 & 4 & 4 & 4 & 4 & 4 & 3 & 4 & & & & & 4 \\
\hline Juniperus phoenicea L. & Cupressacées & 0 & 0 & + & 1 & & + & & & & & 2 & & & & 2 \\
\hline Lagurus ovatus $L$. & Poacées & 2 & 0 & & & & & & & & & 1 & & & & 1 \\
\hline Limonium sinuatum (L.) Mill. & Linacées & 0 & 0 & 0 & 0 & 0 & 0 & + & & & & & & & & \\
\hline
\end{tabular}




\begin{tabular}{|c|c|c|c|c|c|c|c|c|c|c|c|c|c|c|c|c|}
\hline Linum strictum L. & Linacées & 3 & 0 & & & & & + & & + & 1 & 1 & & & & \\
\hline Lygeum spartum L. & Poacées & & & & & & & & & & & 1 & & & & \\
\hline Marrubium vulgare L. & Lamiacées & 0 & 0 & 0 & 0 & 0 & + & + & & & & + & & & & 4 \\
\hline Medicago marina L. & Fabacées & 0 & 0 & 0 & 0 & 0 & 0 & 0 & 0 & & 1 & 2 & 2 & + & & 3 \\
\hline Medicago minima Grufb. & Fabacées & 0 & 0 & 0 & 0 & 0 & 0 & 0 & + & & 1 & & & & & \\
\hline Medicago littoralis Rhode. & Fabacées & & & & & & & & & & 1 & & & & & \\
\hline Mesembryanthemum nodiflorum $L$. & Aizoacées & 0 & 0 & 0 & + & & & & & & & & & & & \\
\hline Muscari comosum (L.) Mill. & Liliacées & 0 & 0 & 0 & 0 & 0 & 0 & 0 & + & & & & & & & \\
\hline Myrtus communis $M$. & Myrtacées & 0 & + & & & & + & + & & + & & & & & & \\
\hline Olea europaea $L$. & Oléacées & 0 & 0 & 0 & 0 & 0 & 0 & + & + & & & & & & & \\
\hline Ononis spinosa $L$. & Fabacées & + & 0 & & & & & & & & & & & & & 3 \\
\hline Ononis natrix L. & Fabacées & 0 & 0 & 0 & 0 & + & & & & + & 1 & & + & & 1 & \\
\hline Paronychia argentea (Pourr.) Lamk. & Caryophyllacées & 2 & 0 & 1 & + & & & & & & & & & & & + \\
\hline Phagnalon saxatile (L.) Cass. & Astéracées & 2 & 2 & 3 & 3 & 2 & 3 & + & & + & 2 & & & & & \\
\hline Phragmites communis & Poacées & & & & & & & & & + & & + & & & & + \\
\hline Pinus halepensis L. & Pinacées & 1 & 0 & & & & & & & & & & & 1 & & \\
\hline Pinus maritima $L$. & Pinacées & 2 & 0 & 3 & 1 & & 1 & 4 & 2 & 2 & 1 & & & & & 1 \\
\hline Pistacia lentiscus L. & Oléacées & 0 & 0 & + & + & + & & & & & & & & & & \\
\hline Plantago argentea Desf. & Plantaginacées & 3 & 0 & & & & & & & & & 1 & & & & \\
\hline Plantago lagopus L. & Plantaginacées & & & & & & & & & + & 1 & & & + & 1 & \\
\hline Plantago marina L. & Plantaginacées & 0 & 2 & 2 & & & + & & & & & 1 & & & & \\
\hline Plantago psyllium L. & Fabacées & 0 & 1 & 2 & & & + & & & & & & & & & \\
\hline Quercus coccifera L. & Fagacées & 3 & 2 & 2 & & & & & & & & 3 & & & & \\
\hline Raphanus raphanistrum $L$. & Brassicacées & 0 & 0 & 0 & + & & & & & & & & & & & \\
\hline Reichardia tingitana (L.) Roth. & Astéracées & 0 & 0 & 0 & + & & & & & & & & & & & \\
\hline Rhamnus alaternus L. & Rhamnacées & 0 & 0 & 0 & 0 & 0 & 1 & & & 1 & + & & & & & + \\
\hline Rhamnus lycioides L. & Rhamnacées & 1 & 1 & & & 1 & + & & & 1 & 1 & & & & & \\
\hline Rosmarinus officinalis $L$. & Lamiacées & & & & & & & & & 1 & + & & & & & \\
\hline Rubia peregrina L. & Rubiacées & & & & & & & & & & & & & & & 1 \\
\hline Rubia sp L. & Rubiacées & 0 & 0 & 4 & 4 & & + & & & & & & & & & \\
\hline Salicornia ramosissima $L$. & Chénopodiacées & & & & & & & & & + & + & & & & & \\
\hline Scabiosa slellata L. & Dipsacacées & 2 & 1 & 3 & 2 & 1 & + & & 2 & + & 2 & & & & & + \\
\hline Scorpiurus vermIculatus $L$. & Fabacées & & & & & & & & & 1 & + & & & & & \\
\hline Senecio leucanthemifolius Poiret. & Astéracées & & & + & & & & & & & & + & + & & & \\
\hline Silene coeli-rosa (L.) A. Br. & Caryophyllacées & & & + & & & & & & & & 2 & & & 2 & 1 \\
\hline Silene maritima L. & Caryophyllacées & 2 & 2 & & & 2 & & & & & & & & & & \\
\hline Spartium junceum L. & Fabacées & + & 1 & 3 & 4 & 3 & 3 & & & 1 & 2 & & & & & \\
\hline Teucrium fruticans $L$. & Lamiacées & 0 & 1 & & & & & & & + & 1 & & & & & \\
\hline Teucrium polium L. & Lamiacées & & & & & & & & & + & 1 & + & & & & \\
\hline Thymus ciliatus Desf. & Lamiacées & & & & & & & & & 1 & + & + & & & & 3 \\
\hline Trifolium stellatum L. & Fabacées & 0 & 1 & 0 & + & 1 & & + & & + & 1 & & & + & & \\
\hline Ulex parviflorus Pourret. & Fabacées & 0 & 0 & 0 & 1 & & + & & & & & & & & + & \\
\hline
\end{tabular}


Table 2. Them floristic surveys of beach of Ouled Ben Ayad ( Ghazaouet)

\begin{tabular}{|c|c|c|c|c|c|}
\hline \multicolumn{6}{|l|}{ Station : Ouled Ben Ayed } \\
\hline \multicolumn{6}{|l|}{ exposition : north east } \\
\hline \multicolumn{6}{|l|}{ Covering rates : $40-50 \%$} \\
\hline \multicolumn{6}{|l|}{ Substrate : silicious } \\
\hline Altitude (m) & & 185 & 16 & 40 & 20 \\
\hline GENRES SPECIES & Survey & 1 & 2 & 3 & 4 \\
\hline Alopecurus pratensis $L$. & Poacées & + & & + & \\
\hline Arenaria emarginata Brot. & Caryophyllacées & + & & + & + \\
\hline Asteriscus maritimus (L.) Less. & Astéracées & 1 & + & + & + \\
\hline Atriplex halimus $L$. & Chénopodiacées & + & & & + \\
\hline Avena sterilis $L$. & Poacées & + & & + & + \\
\hline Bromus madritensis $L$. & Poacées & 1 & & + & 1 \\
\hline Bromus rubens $L$. & Poacées & + & & & 1 \\
\hline Cakile maritima Scop. & Brassicacées & + & & + & + \\
\hline Centaurea pullata $L$. & Astéracées & + & + & + & + \\
\hline Chrysanthemum grandiflorum (L.) Batt. & Astéracées & 1 & 1 & + & + \\
\hline Dactylis glomerata L. & Poacées & 1 & & + & 1 \\
\hline Erodium moschatum $L$. & Géraniacées & + & + & + & + \\
\hline Frankenia laevigata $L$. & Frankeniacées & + & + & + & + \\
\hline Gnaphalium luteo-album L. & Astéracées & + & & + & + \\
\hline Hedysarum sp L. & Fabacées & 1 & 1 & 1 & + \\
\hline Hippocrepis multisiliquosa L. & Brassicacées & + & & + & + \\
\hline Hordeum murinum Witth. & Poacées & 1 & 1 & + & + \\
\hline Inula crithmoides $L$. & Astéracées & + & & + & + \\
\hline Lagurus ovatus $L$. & Poacées & 1 & 1 & 1 & + \\
\hline Lavatera maritima Gouan. & Malvacées & 1 & 1 & + & + \\
\hline Lobularia maritima (L.) Desv. & Brassicacées & + & & + & + \\
\hline Lolium rigidum Gaud. & Poacées & + & & + & 1 \\
\hline Lotus ornithopoides $L$. & Fabacées & + & & + & + \\
\hline Malva sylvestris $L$. & Malvacées & + & & + & + \\
\hline Matthiola sinuata (L.) R. Br. & Fabacées & 1 & + & & + \\
\hline Medicago marina $L$. & Fabacées & + & 1 & 1 & + \\
\hline Medicago littoralis Rhode. & Fabacées & + & + & + & + \\
\hline Medicago minima Grufb. & Fabacées & + & & + & \\
\hline Oxalis pes-caprae $L$. & Oxalidacées & + & & + & + \\
\hline Orchis purpurea L. & Orchidacées & + & + & & + \\
\hline Ononis natrix $L$. & Fabacées & & 1 & + & \\
\hline Paronychia argentea (Pourr.) Lamk. & Caryophyllacées & & + & & + \\
\hline Phagnalon saxatile (L.) Cass. & Astéracées & + & & + & + \\
\hline Plantago lagopus L. & Plantaginacées & + & + & & + \\
\hline Plantago marina $L$. & Plantaginacées & + & & + & + \\
\hline Plantago psyllium L. & Plantaginacées & + & 1 & & + \\
\hline Raphanus raphanistrum $L$. & Brassicacées & 1 & 1 & 1 & + \\
\hline Reichardia tingitana (L.) Roth. & Astéracées & + & & + & + \\
\hline Senecio leucanthemifolius Poiret. & Astéracées & + & & + & + \\
\hline Silene maritima $L$. & Caryophyllacées & + & & + & + \\
\hline Suaeda maritima (L.) Dumort. & Chénopodiacées & + & + & & + \\
\hline Trifolium angustifolium $L$. & Fabacées & + & & & + \\
\hline Trifolium stellatum L. & Fabacées & + & & + & \\
\hline
\end{tabular}


Table 3. Them floristic surveys of beach of Beni Saf; Sidi Boucif and Sid Safi

\begin{tabular}{|c|c|c|c|c|c|c|c|c|c|c|c|c|c|c|c|c|c|c|c|c|c|}
\hline \multicolumn{6}{|l|}{ Station : beach of Beni Saf } & \multicolumn{9}{|c|}{ Station : beach of Sidi Boucif } & \multicolumn{7}{|c|}{ Station : beach of Sidi Safi } \\
\hline \multicolumn{6}{|l|}{ Exposition : North } & \multicolumn{9}{|c|}{ exposition : North } & \multicolumn{7}{|c|}{ Exposition : North } \\
\hline \multicolumn{6}{|l|}{ Covering rates: $05-10 \%$} & \multicolumn{9}{|c|}{ Covering rates : $20-25 \%$} & \multicolumn{7}{|c|}{ Covering rates : $10-20 \%$} \\
\hline Altitude (m) & & 190 & 209 & 212 & 160 & 191 & 191 & 200 & 200 & 210 & 210 & 210 & 200 & 200 & 271 & 273 & 270 & 260 & 215 & 202 & 180 \\
\hline GENRES SPECIES & Survey & 1 & 2 & 3 & 4 & 5 & 6 & 7 & 8 & 9 & 10 & 11 & 12 & 13 & 14 & 15 & 16 & 17 & 18 & 19 & 20 \\
\hline Andropogan hirtus $L$. & Poacées & + & & + & 1 & & & & & & & & & & & & & & & & \\
\hline Anagallis arvensis $L$. & Primulacées & & & & & & & & & & & & & & & + & & + & & + & \\
\hline Arenaria emarginata Brot. & Caryophyllacées & 1 & + & & 1 & 1 & & + & + & 1 & 1 & + & + & + & & & & & & & \\
\hline Asparagus stipularis Forsk. & Liliacées & 1 & & + & 1 & 1 & 1 & + & & + & + & & + & & & & & & & & \\
\hline Asteriscus maritimus (L.) Less. & Astéracées & & & & & & + & + & + & + & + & & + & + & 1 & + & 1 & 1 & & 1 & + \\
\hline Atractylis concellata $L$. & Astéracées & + & & & & & & & & & & & & & & + & & & & & \\
\hline Atractylis pycnocephalus $L$. & Astéracées & + & & & & & & & & & & & & & & + & & & & & \\
\hline Atriplex halimus $L$. & Chénopodiacées & & & & + & & & & & & & & & & & & & + & & & \\
\hline Avena sterilis $L$. & Poacées & & & & & + & & & & & + & & + & & & & & & & & \\
\hline Bellis annua $L$. & Astéracées & & & & & + & + & + & + & + & + & & & & & & & & & & \\
\hline $\begin{array}{l}\text { Bupleurum protractum } \\
\text { Hoffm . et Link. }\end{array}$ & Apiacées & 1 & & 1 & + & & & & & & & & & & & & & & & & \\
\hline Bromus rubens $L$. & Poacées & & & & & + & & & & & + & & & & & & & 1 & & & \\
\hline Calycotome spinosa (L.) Link. & Fabacées & + & & + & & & & & & & & & & & & + & & & & & \\
\hline Calystegia soldanella L. & Astéracées & 1 & & + & + & & & & & & & & & & & & & & & & \\
\hline Catananche coerula L. & Astéracées & 1 & + & & + & + & + & + & + & + & + & + & & + & & & & & & & \\
\hline Centaurea pullata L. & Gentianacées & & & & & & + & & & & & & & & & & & & & + & \\
\hline Chamaerops humilis L. & Chénopodiacées & + & & + & 1 & & & & & & & & & & 3 & + & & & & + & 1 \\
\hline Chenopodium album $L$. & Astéracées & & & & & & & & & & & & & & + & & & & & & \\
\hline $\begin{array}{l}\text { Chrysanthemum } \\
\text { grandiflorum (L.) Batt. }\end{array}$ & Astéracées & & & & & & & & & & & & & & + & & & & & & \\
\hline Chrysanthemum coronarium $L$. & Cistacées & + & & 1 & & & & & & & & & & & & & & & & & + \\
\hline Cuscuta sp (Tourn). L. & Cuscutacées & & & & & & + & & & & & & & & & & & & & & + \\
\hline Dactylis glomerata L. & Poacées & & & & & + & + & + & & & & & & & & & & & & & \\
\hline $\begin{array}{l}\text { Daucus carota subsp } \\
\text { gummifer Lamk. }\end{array}$ & Apiacées & 1 & & 1 & + & & & & & & & & & & + & 1 & & 2 & 1 & & 1 \\
\hline Delphinium peregrinum $L$. & Renonculacées & & & & & & & & & & & & & & + & & + & & & & \\
\hline Echinophora spinosa L. & Apiacées & 1 & & + & + & & & & & & & & & & & & & & & & \\
\hline Echium vulgare Tourn. & Borraginacées & & & & & & & & & & & & & & & & 1 & & & & \\
\hline Ephedra fragilis Desf. & Ephedracées & & & & & & & & & & & & & & + & 1 & & & & & \\
\hline Erica arborea $L$. & Ericacées & + & & & & & & & & & & & & & & & & & & & \\
\hline Erica multiflora $L$. & Ericacées & 1 & + & & + & & 1 & + & & + & + & & & + & + & & & & & & \\
\hline $\begin{array}{l}\text { Erodium moschatum } \\
\text { (Burm) L'Her. }\end{array}$ & Géraniacées & & & & & & & & & & & & & & & & & & & & \\
\hline Eryngium tricuspidatum $L$. & Apiacées & & & & & + & & + & + & + & & + & + & + & & & & & & & \\
\hline Eryngium maritimum L. & Apiacées & & & & & & & & & & & & & & & & & & & & 1 \\
\hline Fagonia cretica L. & Zygophyllacées & & & & & & & & & & & & & & + & & & & & & \\
\hline Globularia alypum L. & Globulariacées & & & & & & & & & & & & & & 2 & + & & 3 & + & 1 & 1 \\
\hline Gnaphalium luteo-album $L$. & Astéracées & & & & & + & & + & + & + & + & & + & + & & & & & & & \\
\hline $\begin{array}{l}\text { Halimium halimifolium } \\
\text { (L.) Willk. }\end{array}$ & Cistacées & & & & & & & & & & & & & & 1 & & & & 1 & 1 & + \\
\hline Hedysarum sp L. & Fabacées & 2 & 3 & 1 & + & & & & & & & & & & & & & & & + & \\
\hline Hordeum murinum Witth. & Poacées & & & & & + & & & & + & & & & & & & & & & & \\
\hline Inula crithmoides $L$. & Astéracées & & 1 & & & & & & & & & & & + & & & & & & & \\
\hline
\end{tabular}




\begin{tabular}{|c|c|c|c|c|c|c|c|c|c|c|c|c|c|c|c|c|c|c|c|c|c|}
\hline Juncus maritimus Lamk. & Juncacées & & & & & & & & & & & & & & 2 & 1 & & & & & \\
\hline Juniperus phoenicea $L$. & Cupressacées & 1 & 2 & 2 & 1 & + & & + & & 1 & & & & & 2 & 2 & & & & 1 & \\
\hline Lagurus ovatus $L$. & Poacées & & & & & & & & & & & & & & 1 & + & + & & 2 & & 1 \\
\hline Lavandula stoechas $L$. & Lamiacées & & & & & + & & & & + & & & & + & & & & & & & \\
\hline Lavandula dentata $L$. & Lamiacées & 1 & + & + & 1 & & & & & & & & & & & & & & & & \\
\hline Limonium sinuatum (L.) Mill. & Plumbaginacées & & & & & & & & & & & & & & + & & + & & & 1 & + \\
\hline Linum strictum $L$. & Linacées & & & & & & & & & & & & & & & & & + & & & \\
\hline Lobularia maritima (L.) Desv. & Brassicacées & + & 1 & & + & & & 1 & & & & & & + & & & & & & & \\
\hline Lotus ornithopoides $L$. & Fabacées & 1 & + & & 1 & & & & & & & & & & & & & & & & \\
\hline Malva sylvestris $L$. & Malvacées & & & & & & & & & & & & & & & & & 1 & & & \\
\hline Marrubium vulgare $L$. & Lamiacées & & & & & & & & & & & & & & 3 & 3 & & & 1 & & \\
\hline Medicago marina L. & Fabacées & & & & & & & + & + & + & + & + & & + & & & 1 & 1 & & 1 & \\
\hline Medicago littoralis Rhode. & Fabacées & & & & & & & + & + & + & + & + & & + & & & & & & & \\
\hline $\begin{array}{l}\text { Mesembryanthemum } \\
\text { nodiflorum } L .\end{array}$ & Aizoacées & 1 & + & & + & & & & & & & & & & & & & & & & \\
\hline Ononis spinosa $L$. & Fabacées & & & & & & & & & & & & & & 1 & & & & + & & \\
\hline Periploca laevigata Auct. & Asclépiadacées & & & & & & & & & & & & & & & + & 1 & 1 & & & \\
\hline $\begin{array}{l}\text { Paronychia argentea } \\
\text { (Pourr.) Lamk. }\end{array}$ & Caryophyllacées & & & & & + & & + & + & + & + & & + & + & + & & & & & & \\
\hline $\begin{array}{l}\text { Phagnalon saxatile } \\
\text { (L.) Cass. }\end{array}$ & Astéracées & 1 & + & + & 2 & & & & & & & & & & & & & & & & \\
\hline Pinus maritima $L$. & Pinacées & & & & & & & & & & & & & & 1 & + & & & & & + \\
\hline Pistacia lentiscus L. & Oléacées & 1 & & 2 & 1 & & & & & & & & & & + & + & & + & & & \\
\hline Plantago argentea Desf. & Plantaginacées & & & & & & & & & & & & & & + & & 1 & & & & \\
\hline Plantago marina $L$. & Plantaginacées & 1 & & + & & & & & & & & & & & 1 & 1 & & + & 1 & 2 & \\
\hline Plantago psyllium L. & Plantaginacées & & & & & & & + & + & + & + & & & & & & & & & & \\
\hline Raphanus raphanistrum L. & Brassicacées & & & & & 1 & + & + & + & + & + & + & + & + & & + & & 1 & & & + \\
\hline Reseda alba $L$. & Résédacées & & & & & & & & & & & & & & & & & & & & + \\
\hline $\begin{array}{l}\text { Reichardia tingitana } \\
\text { (L.) Roth. }\end{array}$ & Astéracées & & & & & + & & + & + & + & + & + & + & + & & & & & & & \\
\hline Reseda lutea $L$. & Résédacées & + & & + & + & & & & & & & & & & 1 & & & & + & & \\
\hline Rhamnus alaternus $L$. & Rhamnacées & & & & & & & & & & & & & & & & & 1 & 2 & & + \\
\hline Rhamnus lycioides $L$. & Rhamnacées & & & & & + & & & & & + & & + & $*$ & & & & & & & \\
\hline Rosmarinus officinalis $L$. & Lamiacées & + & 1 & & 1 & & & & & & & & & & & & & & & & \\
\hline Rubia peregrina L. & Rubiacées & + & & + & & & & & & & & & & & & & & & & & \\
\hline Rumex bucephalophorus $L$. & Polygonacées & 1 & & 1 & + & + & + & & & & + & + & & & & & & & & & \\
\hline Ruta chalepensis L. & Rutacées & & & & & & & 1 & 1 & 1 & + & & + & + & & & & & & & \\
\hline Salicornia ramosissima $L$. & Chénopodiacées & & & & & & & & & & & & & & & & & + & & & 1 \\
\hline Satureja graeca L. & Lamiacées & + & 1 & 1 & & & & & & & & & & & & & & & & & \\
\hline Scorpiurus vermIculatus $L$. & Fabacées & & & & & & & & & & & & & & + & & & 3 & 1 & & \\
\hline Sedum acre $L$. & Crassulacées & + & & 1 & & + & & + & + & + & + & + & + & + & & & & & & & \\
\hline Senecio leucanthemifolius & Astéracées & & & & & & & & & & & & & & 1 & & & 1 & & & \\
\hline Poiret. & & & & & & & & & & & & & & & & & & & & & \\
\hline Silene maritima $L$. & Caryophyllacées & & & & & & & & & & & & & & 3 & 2 & & 1 & 2 & 1 & 1 \\
\hline Smilax aspera $L$. & Liliacées & & & & & & & & & & & & & & 4 & 3 & + & & + & 3 & 4 \\
\hline Stipa tortilis Desf. & Poacées & & & & & & + & & & & & + & & & & & & & & & \\
\hline Taraxacum officinalis $L$. & Astéracées & + & & + & & + & & & + & + & + & + & + & & + & & & & 1 & + & + \\
\hline Tamarix gallica L. & Tamaricacées & & & & & & & & & & & & & & & & & + & & 1 & \\
\hline Teucrium polium $L$. & Lamiacées & + & 1 & + & 1 & & & & & & & & & & & 3 & & + & 1 & 1 & 1 \\
\hline Trifolium stellatum L. & Fabacées & & & & & & & & & & & & & & 2 & 1 & & & & + & 1 \\
\hline Ulex parviflorus Pourret. & Fabacées & & & & & & & & & & + & + & + & & & & & & & & \\
\hline
\end{tabular}




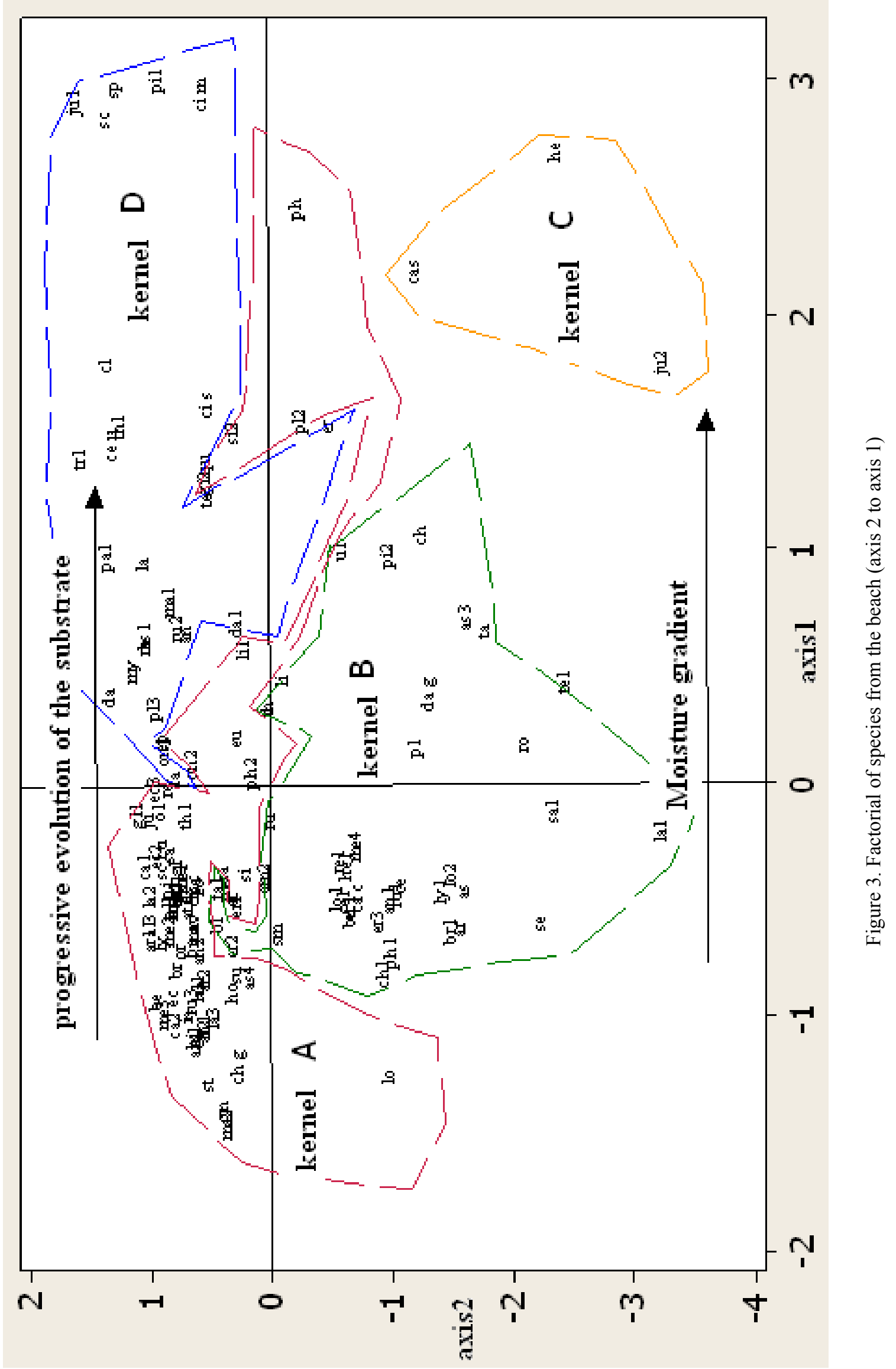




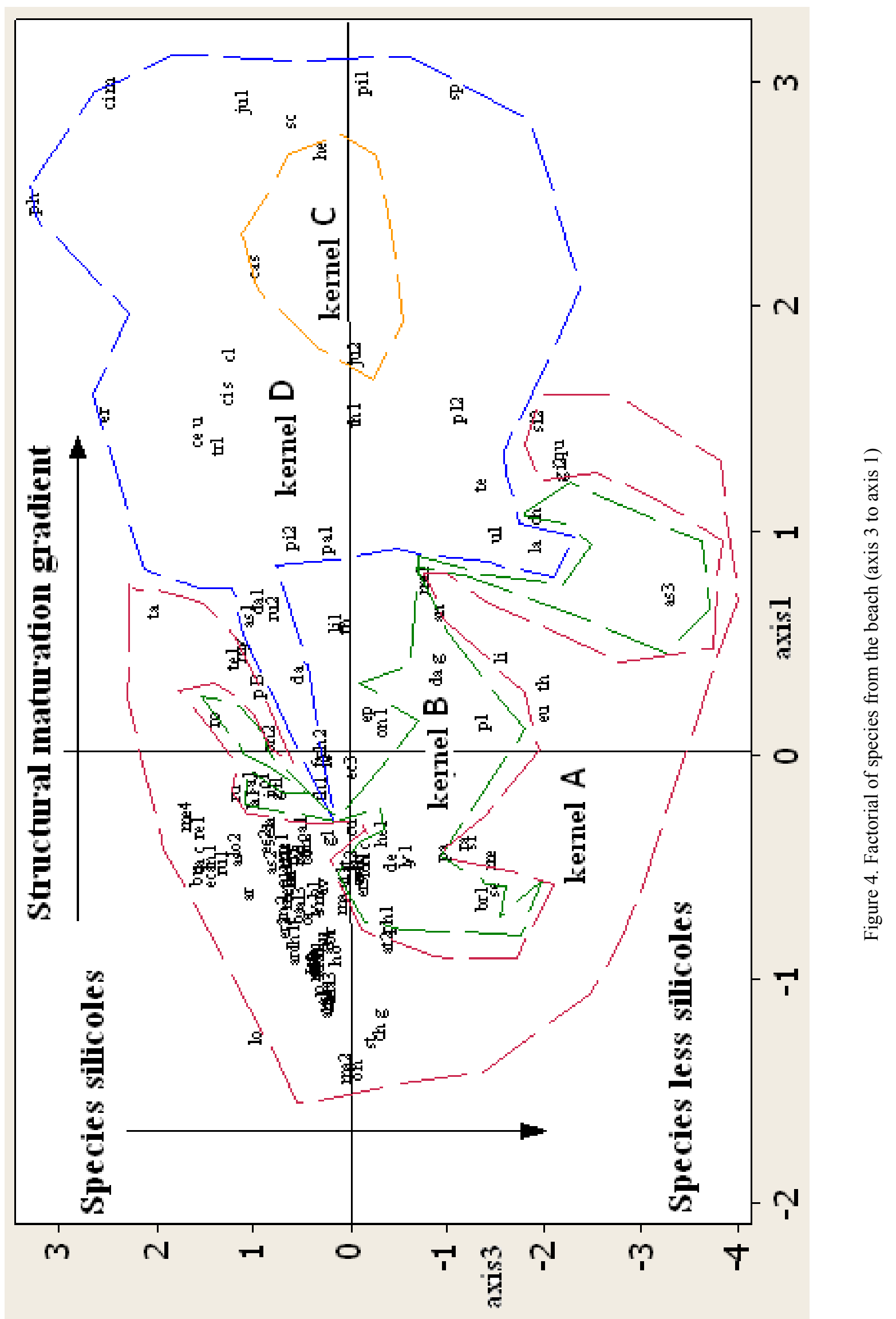




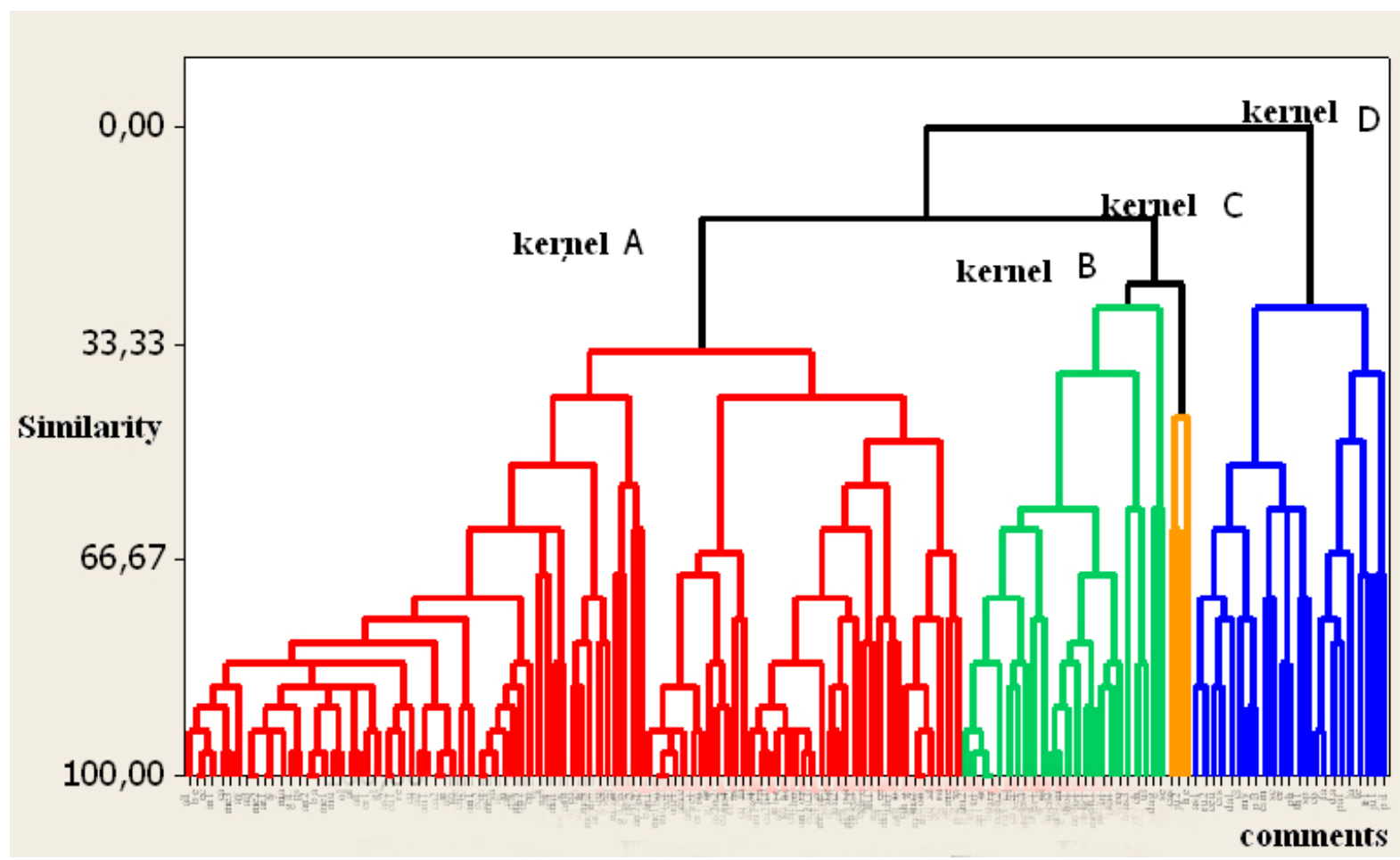

Figure 5. Dendrogram of the species from the beach

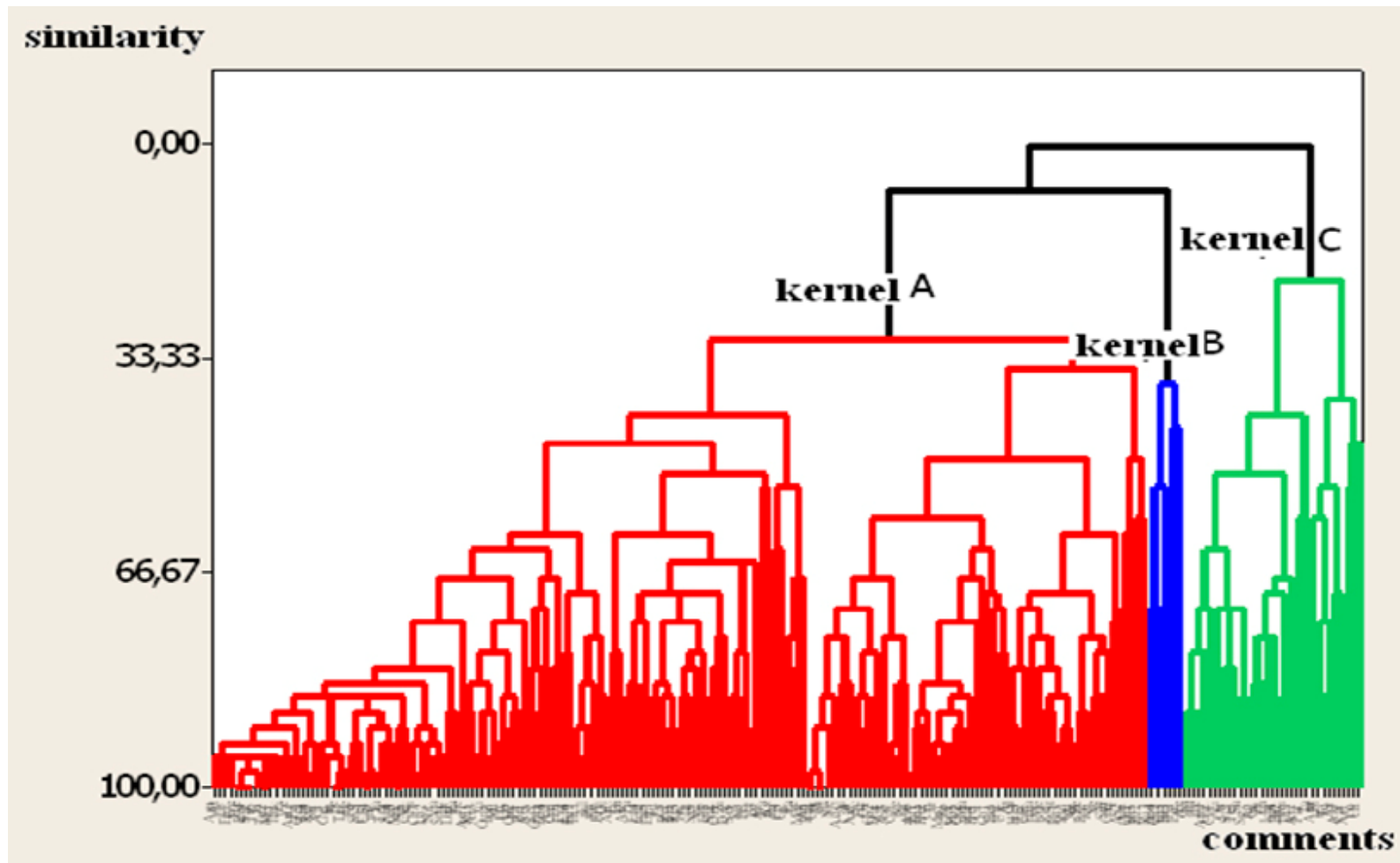

Figure 6. Dendrogram of coastal species 

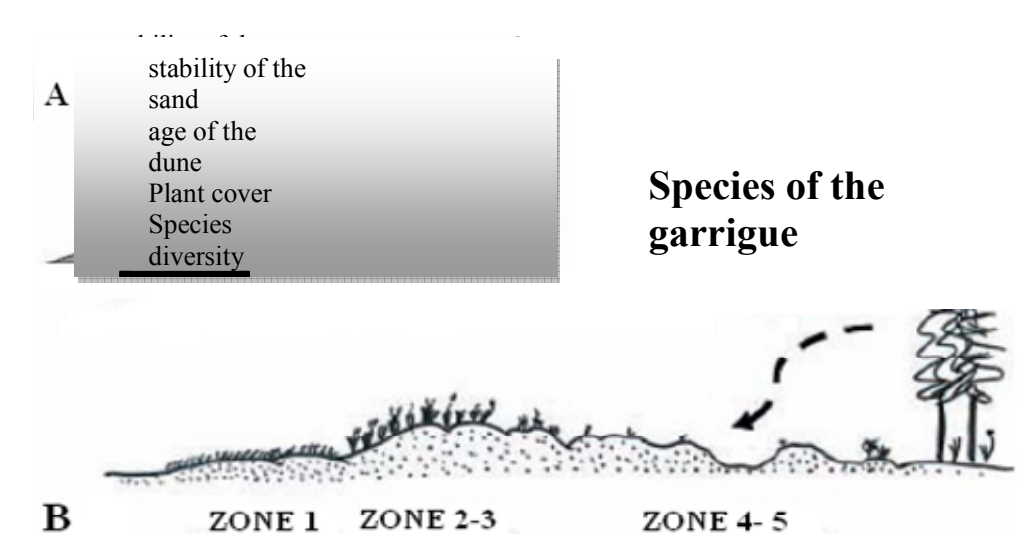

B

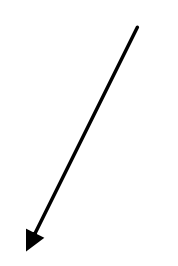

Agropyretum mediterraneum Agropyrum junceum Cyperus aegyptiacus

maritimum.

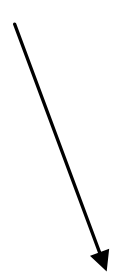

L'Ammophiletum

- Ammophila arenaria

-Medicago marina

- Echinophora spinosa

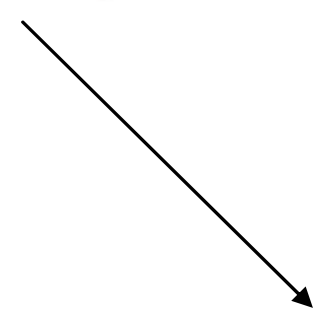

- Crucianella maritima

- Medicago littoralis

- Pancratium

C embryo Dune

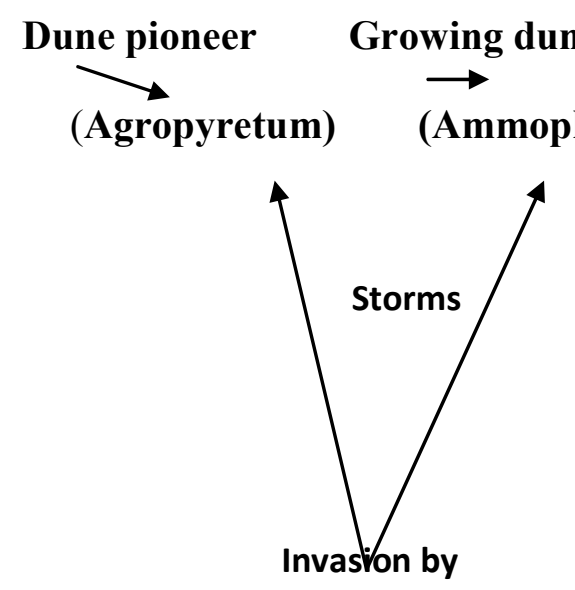

Sand bare

$\underset{\longrightarrow}{\text { Growing dune }}$

hiletum)

Crucianelletum

$\nearrow_{\text {Forest }}$

Ammophila

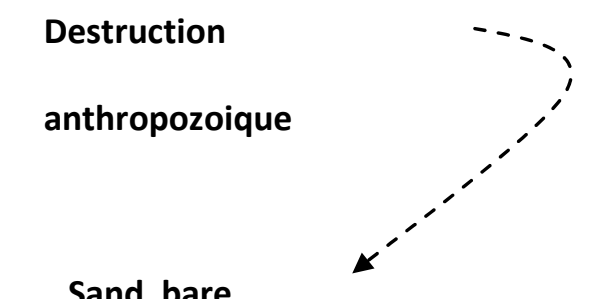

= erosion

Figure 7. Schematic summary of the ecology of sandy coastline. (KUNHOLTZ - LORDAT 1923)

A: Appearance of the beach and summary of the reciprocal action of physical factors and vegetation. B: Vegetation Associations typical of different areas of the beach, with their characteristic species. These species are typically arranged in their order of appearance. C: Succession of the main plant associations of the sandy coastline. Disturbance (antropozoogenes or natural, tillé line arrows) cause regression of vegetation. The natural dynamics of the system leads to the establishment of a cyclic process. 


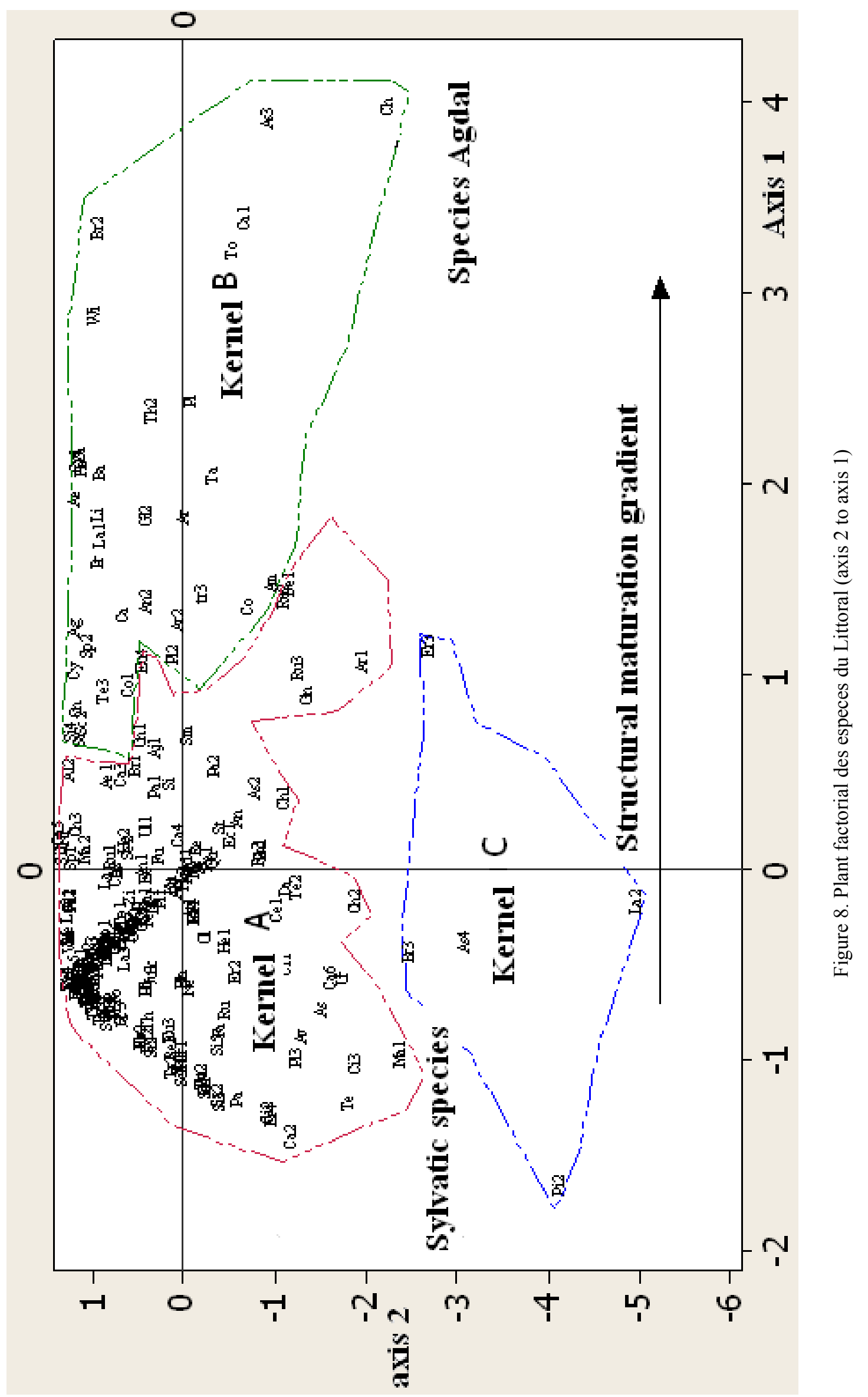




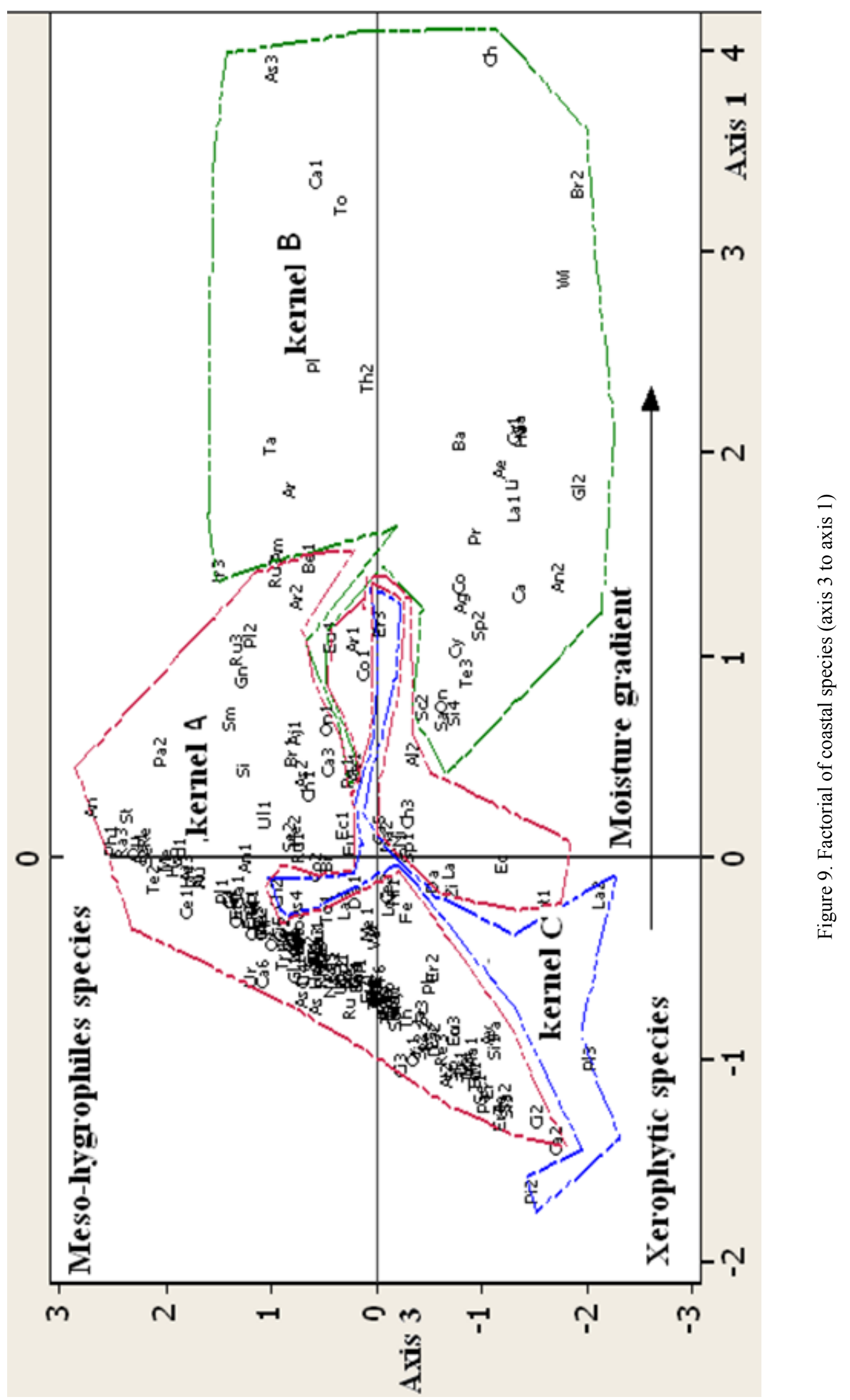




\section{Results and Discussion (of the Species of the Coast)}

It is a set of 57 phytosociological surveys and 223 species on the sandy coast of Sid Safi station; Rechgoune and Ghazaouet.

On the coast, the majority of the species belonging to the class of the Therobrachypodietea

\subsection{Plan 2/1:}

Negative side: Ammoides verticillata, Arisarum vulgare, Asparagus acutifolius, Asteriscus maritimus, Avena sterilis, Cistus ladaniferus, Cistus monspeliensis, Eryngium maritimum, Eryngium tricuspidatum, Marrubium vulgare, Lobularia maritima, Genista numidica, Pistacia lentiscus, Tetraclinis articulata

Positive side: Althaea hirsuta, Atractylis carduus, Carthamus coerulus, Centaurea pungens, Retama monosperma In this area we find species very opposed by their procession and their vocation. The first batch consists of pre-stock species where a semblance of woodsy atmosphere. The second batch of so-called species mate without any taxonomic convergence

4.2 Plan $3 / 1$ :

Negative side: Aegilops ventricosa, Agropyron repens, Anthyllis tetraphylla, Avena sterilis, Atractylis concellata, Prasium majus, Plantago lagopus, Pistacia lentiscus, Tetraclinis articulata, Teucrium pseudo-chamaepitys, Echinops spinosus, Eryngium tricuspidatum

Positive side: Trifolium compestre, Teucrium polium, Stipa tenacissima, Smilax aspera, Raphanus raphanistrum, Reichardia picriodes, Reichardia tingitana, Quercus coccifera, Phylleria angustifolia, Ophrys apifera, Orchis coriophora, Medicago littoralis

On the positive side there are relatively meso hygrophilous such as species Ophrys apifera; Orchis coriophora . and the negative side are relatively more tolerant species: Echinops spinosus ; Eryngium tricuspidatum; Atractylis concellata

This very clear axis to identify by a moisture gradient. Values are so low, they are difficult to interpret. The structuring of the cloud is no worse; Add to this the human conversations at this level leading to homogenization of the flora.

This region corresponds to a fixed dune characterized by a grouping evolved; plastic and weakly psammophilous, differentiated by Juniperus phoenicea, Pinus maritime, Erica multiflora, Asparagus acutifolius, Asparagus stipularis.

This advanced considered grouping formed the coastal juniperais. On the site map, it is aimed at altitudes of $100 \mathrm{~m}$ to $400 \mathrm{~m}$, on slopes ranging from 5 to $25 \%$ and forming a collection of $70-90 \%$; It is noted from the wet to the semi-arid.

The presence of this group of species is explained by its spatial heterogeneity and has his adaptation (R strategies). This plasticity confirms it a wider environmental spectrum, and colonization of the dunes by producing many seed species (Pinus halepensis; Juniperus phoenicea).

\section{Conclusion}

The analysis of the A.F.C highlighted 03 vegetal groups that organise themselves on the map $2 / 1$ and $3 / 1$ according to a schema corresponding to the analysis of adaptive strategies (MAC-ARTHUR 1957) in Chaabane (1993). This segregation is a variation of soil moisture and textural and structural elements. Furthermore, Therophytiques nitrophilous species endowed with a strong potential biotic and reciprocal growth settled more easily, there will be designated the R selection, the form's own selection.

The three groupings sets are represented by:

Groups psammophilous by excellence:

Ammophila arenaria, Cakile maritima, Calystegia soldanella, Eryngium maritimum, Medicago minima, Medicago marina

These species pertaining to Ammophiletea and Cakiletea maritimae class. They occupy the vertices of the beaches in maritime borders and also it means vegetation psammo-halo-nitrophilous therophytes (Chaabane, 1993) that characterize the embryonic dunes.

In moving away from the beach, a very diverse vegetation moved to attach these dunes giving birth to more or less fixed bright dunes. This vegetation is related to the Therobrachypodietea class 
The interaction of different natural factors has the mosaics of biotopes and vegetation structure. But this arrangement, linked to the variability of the physicochemical characteristics of the substrates, is particularly disturbed by the actions of origins anthropozoogenic.

This pressure results in depletion of the most advanced sets, a loss of 'natural' biodiversity of specific groups of the coast; it caused great difficulty in the individualization says beaches (Cakiletea maritimae) or even of dunes fixed sets.

Finally, we say that the future is worrying about maintaining this national heritage. It is important that effective, even drastic measures be taken rapidly to alleviate this pressure of anthropozoic origin. Admittedly, this is not unique to the Algerian coast since it occurs in all countries of the Maghreb and on almost all of the territories, but the reduction of vested coastal areas to natural ecosystems is an aggravating factor.

It is with this concern to reduce the pressure, or even to improve the forest of the spit, that we brought the attention on Ammophila arenaria.

This species by their leaf system, its flexibility and its flexibility slows the speed of the wind and allow sand accumulation. It has good resistance to loosening and stabilizes the dunes by its root systems. Lay down the dunes and Ammophila arenaria in the coast plantations offer a picturesque great heritage value and original topography.

\section{References}

Bouazza, M., \& Benabadji, N. (1998). Composition floristique et pression anthropozö̈que au Sud-Ouest de Tlemce (pp. 93-97). Rev. Sci. Tech. Univ. Constantine, Algérie.

Chaabane, A. (1993). Etude de la végétation du littoral septentrional de Tunisie: typologie, syntaxonomie et éléments d'aménagement (Doctoral dissertation). Es-sciences en Ecologie. Uni. Aix-Marseille III

Dahmani-Megrouche, M. (1997). Le chêne vert en Algérie. Syntaxonomie phytosociologie et dynamique des peuplements (Doctoral dissertation, Thèse doct. Es-sciences. UnivHouariBoumediene. Alger).

Favennec, J. (2002). Guide de la flore des dunes littorales de la Bretagne au Sud des Landes. Edition sud ouest/ONF.

Guardia, P. (1975). Géodynamique de la marge alpine du continent africain d'après l'étude de l'Oranie nord-occidentale: relations structurales et paléogéographiques entre le Rif externe, le Tell et l'avant-pays atlasique (Doctoral dissertation). Thèse 3ème cycle, Univ. Nice.

Kuhnholtz-Lordat, G. (1923). Les dunes du Golfe du Lion: (essai de géographie botanique). Paris: Les Presses Universitaire de France.

Quézel, P. (2000). Réflexions sur l'évolution de la flore et de la végétation au Maghreb méditerranéen (Ibis Press Edit). Paris: Ibis Press.

Zeraia, L. (1981). Essai d'interprétation comparative des données écologiques, phénologques et de production subero-ligneuse dans les forêts de chêne-liège de Provence cristalline (France méridionale) et d'Algérie (Doctoral dissertation).

\section{Copyrights}

Copyright for this article is retained by the author(s), with first publication rights granted to the journal.

This is an open-access article distributed under the terms and conditions of the Creative Commons Attribution license (http://creativecommons.org/licenses/by/3.0/). 